\title{
CircPVT1: a bridge linking Hippo pathway and human cancers
}

\author{
Xiaoyun $\mathrm{He}^{1,2 \#}$, Weiping Su${ }^{3 \#}$, Yangying Zhou ${ }^{4}$, Xiaolu Ge ${ }^{1}$, Jianhua Zhou ${ }^{1}$, Chunlin $\mathrm{Ou}^{1}$ \\ ${ }^{1}$ Department of Pathology, ${ }^{2}$ Department of Endocrinology, ${ }^{3}$ Department of Orthopedics, ${ }^{4}$ Department of Oncology, Xiangya Hospital, Central \\ South University, Changsha 410008, China \\ \#These authors contributed equally to this work. \\ Correspondence to: Chunlin Ou; Jianhua Zhou. Department of Pathology, Xiangya Hospital, Central South University, Changsha 410008, China. \\ Email: ouchunlin@csu.edu.cn; zhoujh15@163.com. \\ Comment on: Verduci L, Ferraiuolo M, Sacconi A, et al. The oncogenic role of circPVT1 in head and neck squamous cell carcinoma is mediated \\ through the mutant p53/YAP/TEAD transcription-competent complex. Genome Biol 2017;18:237.
}

Submitted Oct 30, 2018. Accepted for publication Nov 01, 2018.

doi: $10.21037 / \mathrm{atm} .2018 .11 .06$

View this article at: http://dx.doi.org/10.21037/atm.2018.11.06

The Hippo pathway, first discovered in Drosophila (1), plays an important role in regulating the size of tissues and organs, and maintains homeostasis by modulating cell proliferation and apoptosis. The Hippo pathway itself is regulated by intrinsic cell signals, such as cell polarity, cellcell contact, and by the actin cytoskeleton (2). A critical downstream effector of the Hippo pathway-YAP is reported to be highly expressed in many solid tumors, where it promotes tumor cell proliferation, invasion/migration, and maintenance of stemness of cancer cells (3). YAP not only acts as a transcriptional modulator in the Hippo signaling pathway (4), but also acts as a link between the Hippo/YAP pathway and other signaling pathways, thereby regulating the biological functions (5). The signaling pathways linked to the Hippo pathway are G-Protein-Coupled Receptor (GPCR) (6), NF- $\kappa B$ (7), Wnt/ $\beta$-catenin (8), TGF- $\beta /$ SMAD (9), epidermal growth factor receptor (EGFR) (10), and Notch signaling pathways (11). These signaling pathways form a complex regulatory network, with YAP as its core and interact with different molecules involved in various molecular cascades, thereby regulating the progression and development of cancers. However, recent studies have revealed that YAP plays a dual-role in many aspects of tumor development, which includes controlling the tumorigenicity of cancer cells, regulating stem cell differentiation and inflammatory reaction, survival, and prognosis. Therefore, we performed a meta-analysis study on 2,983 cases of different malignant tumors (obtained from 21 independent studies), in which there was an upregulation of YAP1 and reduced overall survival/ disease- free survival of tumor patients, suggesting that YAP1 is not conducive for prognosis (12).

YAP acts as a transcriptional co-activator since it cannot bind to DNA directly. Therefore, YAP interacts with DNA-binding transcription factors (e.g., TEAD14, RUNX1/2, Smad, and p63/p73, mutated p53) and controls transcriptional regulation. For example, YAP directly induces miR-130a that could effectively repress the inhibitor of YAP VGLL4, thereby forming a positive feedback loop to amplify the YAP signal, which plays a crucial role in controlling organ size during development and tumorigenesis in human cancers (13). Another report suggested that UCA1 interacted with Lats1, MOB1, and YAP, forming a complex, thereby increasing YAP nuclear localization and stabilization. The nuclear-localized YAP interacts with a transcription factor TEAD1 to promote UCA1 expression, indicating the presence of a "YAP1UCA1" axis that promotes cell migration and invasion in pancreatic cancer (14). Many studies have suggested that YAP can regulate the processing of miRNA and lncRNA (15); however, the interaction of YAP-CircRNAs (circular RNAs) and their biological function in tumorigenesis remain largely unknown.

CircRNAs is a new class of non-coding RNAs, and their length exceeds 200 nucleotides. CircRNAs, unlike linear RNAs, are formed by a covalently closed loop that lacks the $5^{\prime}-3$ ' ends and the poly A tail (16) that makes them comparatively more stable and resistant to degradation by RNase R or RNA exonuclease (17). CircRNAs play a critical role in regulating physiological and pathological functions 
in humans. Physiological functions include stem cell selfrenewal, cell proliferation, and apoptosis; whereas, an aberrant expression of circRNAs leads to various diseases, including tumors (18). In the past decade, circRNAs have gained widespread attention. A literature search of PubMed, Embase, and Web of Science was performed using the following search terms: "CircRNA" or "Circular RNAs" AND "disease." After excluding case reports, meta-analyses, reviews, comments, letters, and duplicate publications, a total of 163 papers were found on the relationship between circRNAs and diseases, by the end of June 22, 2018. Since 2015, the number of relevant publications has been increasing rapidly, indicating that researchers are showing a fast-growing interest towards the functions of CircRNAs in human diseases. Moreover, several websites have been developed as convenient tools to catalog data collected from genomic analyses conducted across multiple labs, including CSCD (cancer-specific circRNAdatabase) (http:// gb.whu.edu.cn/CSCD), PlantcircBase (http://ibi.zju.edu.cn/ plantcircbase/), circInteractome (https://circinteractome. nia.nih.gov/), circRNADisease (http://cgga.org.cn:9091/ circRNADisease), circRNAdb (http://reprod.njmu.edu. cn/circrnadb), CircNet (http://circnet.mbc.nctu.edu.tw/), circBase (www.circbase.org) and circ2 Traits (http://gyanxetbeta.com/circdb/).

CircPVT1, also known as circ6 (16), is a homologous gene of the long non-coding RNA PVT1 (human genome GRch38/hg38) and is generated from exon 2 of the PVT1 gene. CircPVT1 has been widely studied in recent years, and is reported to be up-regulated in many types of cancers, including osteosarcoma (19), ALL (acute lymphoblastic leukemia) (20), and gastric cancer (21). These studies have revealed that CircPVT1 is associated with cancer cell proliferation, invasion, and metastasis. Interestingly, CircPVT1 is a senescence-associated circRNA exhibiting elevated levels of expression in dividing cells and reduced levels in senescent fibroblasts, thereby inhibiting cell senescence and promoting cell proliferation (22). The most important function of CircPVT1 is their activity as miRNA molecular "sponges", thereby increasing the expression of miRNA target genes such as let-7 (22) and miR-125 (21) at the post-transcriptional level. However, the upstream mechanism of CircPVT1 in the progression of tumors remains largely unknown.

In a recent publication (23), Verduci and co-authors from Blandino's group at the Italian National Cancer Institute elegantly demonstrated the role of the circPVT1 in head and neck squamous cell carcinoma (HNSCC). The authors showed that CircPVT1 was up-regulated and associated with the TP53 mutations in HNSCC. It could serve as an independent risk factor for monitoring the course of HNSCC. In HNSCC, CircPVT1 is transcriptionally enhanced by the mut-p53/YAP/TEAD1 complex, which is a continuity work benefiting from the previous study by Blandino group (24) that demonstrated the mutant p53 should be added together the proteins binding YAP. Further, CircPVT1 acts as miRNA molecular "sponge" and binds with miR-497-5p allowing the expression of Aurka, mki67, and bub1 genes, which are associated with cancer cell proliferation, thereby regulating the proliferative phenotype of HNSCC. The authors demonstrated that the "mut-p53/YAP/TEAD1-CircPVT1-miR-497-5p/Aurka/ mki67/bub1" axis plays an important role in promoting the proliferation of HNSCC. Hence, targeting YAP-CircPVT1 may provide a new perspective in designing strategies for the treatment of HNSCC. However, these conclusions need to be validated for the following reasons. 1 Firstly, the author should perform a luciferase assay to verify that miR-497-5p targets the 3' UTR of Aurka/mki67/bub1. Moreover, I consider that the lack of animal models to test their findings has limited the article from being published in a journal with higher impact factor.

In summary, Verduci et al. (23) have clearly established the regulatory loop "YAP-CircPVT1" that regulates cell proliferation in HNSCC (Figure 1). The study has not only expanded our knowledge of the YAP protein, but has also provided new insight into the potential use of "YAP-CircPVT1" for the development of new treatment strategies for HNSCC. Recent studies showed that YAP is a potential target for small-molecule therapeutics, and some therapeutic benefit has been achieved from YAP inhibition in cancer. Recently, a series of small-molecule modulators of YAP have been used in clinical trials, such as, C19 (25), XMU-MP-1 (26), Dobutamine (27), and Verteporfin (28). Besides, the regulatory model of "YAP-ncRNAs" will open a new window into the development of novel therapeutics and diagnostic for the treatment of cancers; although, the underlying molecular mechanism remains largely unknown. We need to further investigate the potential molecular mechanisms of the model of YAP-ncRNAs. Meanwhile, there is also an immediate need to identify and validate more number of unknown ncRNAs by the high-throughput RNA deep sequencing (29-32). Hopefully, with all these efforts, targeting "YAP-ncRNAs" will be a promising therapeutic strategy for the treatment of cancer in the future. 


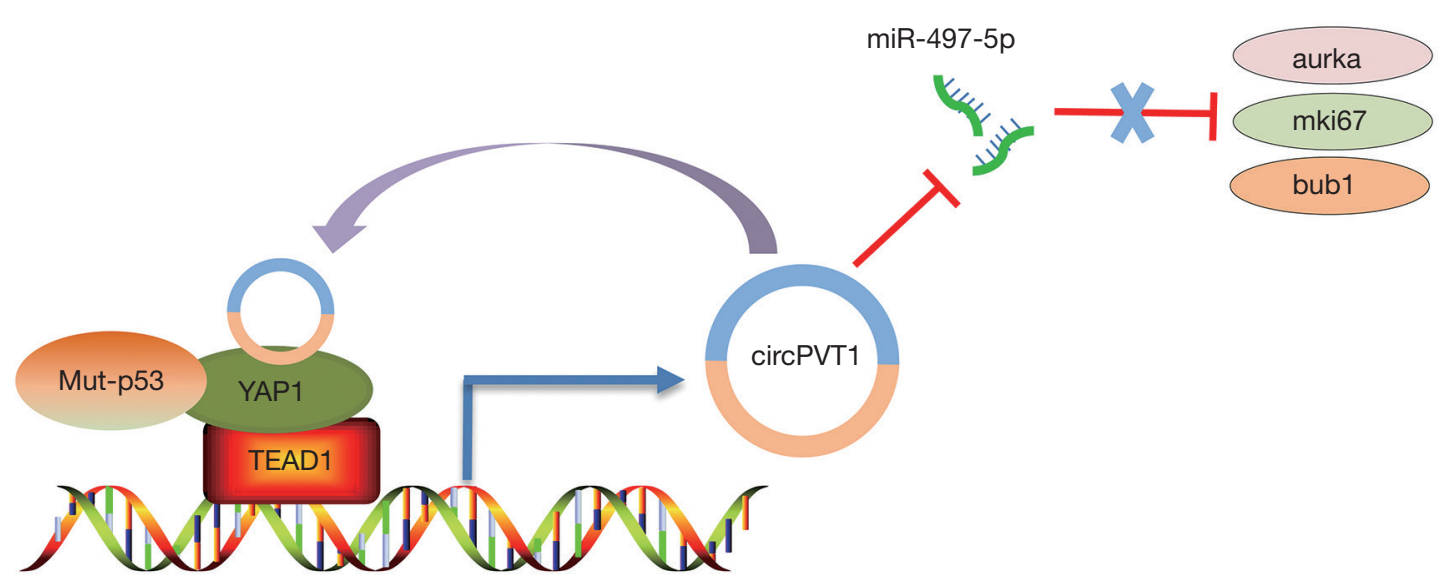

Figure 1 Schematic representation of a model for the "mut-p53/YAP/TEAD1-CircPVT1-miR-497-5p/Aurka/mki67/bub1" axis.

\section{Acknowledgements}

Funding: This work was supported by the National Key Research and Development Program of China (2016YFC1201800), the Natural Science Foundation of Hunan Province of China (14JJ2042) and Central Universities of Central South University (2018zzts044).

\section{Footnote}

Conflicts of Interest: The authors have no conflicts of interest to declare.

\section{References}

1. Harvey KF, Pfleger CM, Hariharan IK. The Drosophila Mst ortholog, hippo, restricts growth and cell proliferation and promotes apoptosis. Cell 2003;114:457-67.

2. Ou C, Sun Z, Li X, et al. MiR-590-5p, a density-sensitive microRNA, inhibits tumorigenesis by targeting YAP1 in colorectal cancer. Cancer Lett 2017;399:53-63.

3. Zhu C, Li L, Zhao B. The regulation and function of YAP transcription co-activator. Acta Biochim Biophys Sin (Shanghai) 2015;47:16-28.

4. Liu AM, Xu MZ, Chen J, et al. Targeting YAP and Hippo signaling pathway in liver cancer. Expert Opin Ther Targets 2010;14:855-68.

5. Ou C, Sun Z, Li S, et al. Dual roles of yes-associated protein (YAP) in colorectal cancer. Oncotarget 2017;8:75727-41.

6. Yu FX, Zhao B, Panupinthu N, et al. Regulation of the Hippo-YAP pathway by G-protein-coupled receptor signaling. Cell 2012;150:780-91.

7. Ou C, Sun Z, Zhang H, et al. SPLUNC1 reduces the inflammatory response of nasopharyngeal carcinoma cells infected with the EB virus by inhibiting the TLR9/NFkappaB pathway. Oncol Rep 2015;33:2779-88.

8. Ou C, Li X, Li G, et al. WWC3: the bridge linking Hippo and Wnt pathways in lung cancer. J Thorac Dis 2017;9:2315-6.

9. Varelas X, Samavarchi-Tehrani P, Narimatsu M, et al. The Crumbs complex couples cell density sensing to Hippodependent control of the TGF-beta-SMAD pathway. Dev Cell 2010;19:831-44.

10. Chaib I, Karachaliou N, Pilotto S, et al. Co-activation of STAT3 and YES-Associated Protein 1 (YAP1) Pathway in EGFR-Mutant NSCLC. J Natl Cancer Inst 2017;109.

11. Tschaharganeh DF, Chen X, Latzko P, et al. Yesassociated protein up-regulates Jagged-1 and activates the Notch pathway in human hepatocellular carcinoma. Gastroenterology 2013;144:1530-42.

12. Sun Z, Xu R, Li X, et al. Prognostic Value of YesAssociated Protein 1 (YAP1) in Various Cancers: A MetaAnalysis. PLoS One 2015;10:e0135119.

13. Shen S, Guo X, Yan H, et al. A miR-130a-YAP positive feedback loop promotes organ size and tumorigenesis. Cell Res 2015;25:997-1012.

14. Zhang $M$, Zhao Y, Zhang $Y$, et al. LncRNA UCA1 promotes migration and invasion in pancreatic cancer cells via the Hippo pathway. Biochim Biophys Acta Mol Basis Dis 2018;1864:1770-82.

15. He X, Yan Q, Kuang G, et al. Metastasis-associated lung adenocarcinoma transcript 1 regulates tumor progression: 
old wine in a new bottle. J Thorac Dis 2018;10:S1088-91.

16. Memczak S, Jens M, Elefsinioti A, et al. Circular RNAs are a large class of animal RNAs with regulatory potency. Nature 2013;495:333-8.

17. Salzman J, Gawad C, Wang P, et al. Circular RNAs are the predominant transcript isoform from hundreds of human genes in diverse cell types. PLoS One 2012;7:e30733.

18. He X, Kuang G, Ou C, et al. Crosstalk between circular RNAs and microRNAs in tumorigenesis. Transl Cancer Res 2017;6:S1448-50.

19. Kun-Peng Z, Xiao-Long M, Chun-Lin Z. Overexpressed circPVT1, a potential new circular RNA biomarker, contributes to doxorubicin and cisplatin resistance of osteosarcoma cells by regulating ABCB1. Int J Biol Sci 2018;14:321-30.

20. Hu J, Han Q, Gu Y, et al. Circular RNA PVT1 expression and its roles in acute lymphoblastic leukemia. Epigenomics 2018;10:723-32.

21. Chen J, Li Y, Zheng Q, et al. Circular RNA profile identifies circPVT1 as a proliferative factor and prognostic marker in gastric cancer. Cancer Lett 2017;388:208-19.

22. Panda AC, Grammatikakis I, Kim KM, et al. Identification of senescence-associated circular RNAs (SAC-RNAs) reveals senescence suppressor CircPVT1. Nucleic Acids Res 2017;45:4021-35.

23. Verduci L, Ferraiuolo M, Sacconi A, et al. The oncogenic role of circPVT1 in head and neck squamous cell carcinoma is mediated through the mutant p53/YAP/ TEAD transcription-competent complex. Genome Biol

Cite this article as: $\mathrm{He} \mathrm{X}, \mathrm{Su}$ W, Zhou Y, Ge X, Zhou J, Ou C. CircPVT1: a bridge linking Hippo pathway and human cancers. Ann Transl Med 2018;6(Suppl 2):S91. doi: 10.21037/ atm.2018.11.06
2017;18:237.

24. Di Agostino S, Sorrentino G, Ingallina E, et al. YAP enhances the pro-proliferative transcriptional activity of mutant p53 proteins. EMBO Rep 2016;17:188-201.

25. Basu D, Lettan R, Damodaran K, et al. Identification, mechanism of action, and antitumor activity of a small molecule inhibitor of hippo, TGF-beta, and Wnt signaling pathways. Mol Cancer Ther 2014;13:1457-67.

26. Fan F, He Z, Kong L, et al. Pharmacological targeting of kinases MST1 and MST2 augments tissue repair and regeneration. Sci Transl Med 2016;8:352ra108.

27. Oudhoff MJ, Freeman SA, Couzens AL, et al. Control of the hippo pathway by Set7-dependent methylation of Yap. Dev Cell 2013;26:188-94.

28. Brodowska K, Al-Moujahed A, Marmalidou A, et al. The clinically used photosensitizer Verteporfin (VP) inhibits YAP-TEAD and human retinoblastoma cell growth in vitro without light activation. Exp Eye Res 2014;124:67-73.

29. Ou C, Li G. Long non-coding RNA TUG1: a novel therapeutic target in small cell lung cancer. J Thorac Dis 2017;9:E644-5.

30. Zhong Y, Du Y, Yang X, et al. Circular RNAs function as ceRNAs to regulate and control human cancer progression. Mol Cancer 2018;17:79.

31. Zhou R, Wu Y, Wang W, et al. Circular RNAs (circRNAs) in cancer. Cancer Lett 2018;425:134-42.

32. Meng S, Zhou H, Feng Z, et al. CircRNA: functions and properties of a novel potential biomarker for cancer. Mol Cancer 2017;16:94. 